\title{
Validation of a Measure of Protective Parent Responses to Children's Pain
}

\author{
Lynn S. Walker, $\mathrm{PhD}^{\star}$, Rona L. Levy, $\mathrm{PhD}^{\dagger}$, and William E. Whitehead, $\mathrm{PhD}^{\ddagger}$ \\ *Vanderbilt University Medical Center \\ tUniversity of Washington \\ ‡University of North Carolina
}

\section{Abstract}

Objectives-To assess the validity of the Protect Scale of the Adult Responses to Children's Symptoms (ARCS) Questionnaire with regard to mothers' responses to their children's abdominal pain.

Methods-Mothers with High $(\mathrm{n}=32)$ and Low $(\mathrm{n}=35)$ Protect scores on the ARCS questionnaire were recruited from participants in a larger study of family illness behavior. Mothers completed a 28-day diary report of responses to their children's abdominal pain episodes. Records of their children's annual health service utilization and costs were obtained from their health maintenance organization (HMO).

Results-Mothers' scores on the ARCS Protect Scale were significantly correlated with their subsequent diary reports of protective responses to their children's abdominal pain. Compared to children of mothers in the Low Protect group, children of mothers in the High Protect group made significantly more health care visits for gastrointestinal symptoms and had significantly higher health care costs.

Conclusions-Results supported the validity of the Protect Scale of the ARCS and demonstrated that mothers' protective responses to children's abdominal pain complaints at home predicted subsequent health service use for gastrointestinal symptoms.

\begin{abstract}
Studies of significant others' behavior toward pain patients have documented the impact of social interactions on pain behavior. For example, solicitous behavior by spouses is associated with increased pain complaints and disability in adults with chronic pain. ${ }^{1-3}$ Similarly, the pediatric literature shows that increased attention from parents is associated with higher levels of pain complaints and disability in children with chronic pain ${ }^{4-7}$ and delayed recovery by children undergoing painful oral surgery. ${ }^{8}$ This research is grounded in social learning theory ${ }^{9}$ and the findings have been interpreted as evidence that significant others may reinforce patients' pain behavior.

Most research in this area uses self-report questionnaires to assess a significant other's typical behavior toward a patient with chronic pain. For example, a subscale of the Multidimensional Pain Inventory asks respondents to rate, on a scale ranging from "never" to "very frequently," how often the patient's spouse engages in solicitous behavior such as taking on the patient's chores. ${ }^{10}$ Respondents must estimate the significant other's "usual" response to the patient's pain. However, to the extent that unique circumstances influence
\end{abstract}

Copyright $\odot 2006$ by Lippincott Williams \& Wilkins

Reprints: Lynn S. Walker, PhD, Vanderbilt University Medical Center (lynn.walker@ vanderbilt.edu).. 
behavior on any given occasion, respondents may find it difficult to describe "usual" behavior. Furthermore, research on self-report methodology suggests that recollections of usual behavior may reflect the individual's expectations rather than actual experience ${ }^{11}$ or may be biased by the most recent episode, the most extreme episode, or other factors associated with particular episodes of a target behavior. ${ }^{12}$ Support for the validity of questionnaire measures of significant others' responses to pain patients would be strengthened by evidence that these measures predict subsequent responses to particular episodes of pain.

In addition to assessing significant others' behavior by means of questionnaires, most research in this area also uses questionnaires to assess patients' pain behavior. Notable exceptions are laboratory studies that assessed patients' verbal pain complaints ${ }^{6}$ or performance on functional tasks such as lifting and walking. ${ }^{2,13}$ Objective measures of pain behavior that occurs outside the laboratory have rarely been obtained. For example, no studies have documented the extent to which questionnaire measures of solicitous parent responding to children's pain correlates with pediatric health service utilization.

The present study assessed the validity of the Protect Scale of the Adult Responses to Children's Symptoms (ARCS) Questionnaire, ${ }^{14}$ a scale that assesses protective parenting in response to children's abdominal pain. First, we evaluated the extent to which mothers' recall of their usual behavior when their children had abdominal pain was associated with mothers' subsequent reports of their behavior in response to specific episodes of abdominal pain assessed during a month-long daily diary study. Because diary methods assess multiple instances of behavior close in time to their actual occurrence, they may have greater reliability and validity than questionnaires and therefore serve as a good standard against which to evaluate the psychometric properties of questionnaires. ${ }^{15}$ Second, we evaluated the extent to which mothers' reports of their usual behavior predicted subsequent health service utilization for their children's abdominal symptoms. We focused on abdominal pain because it is the most common pain complaint in children and adolescents. ${ }^{16} \mathrm{We}$ focused on mothers' protective behavior toward their children because the empirical literature has consistently demonstrated that more protective behavior, referred to as "solicitous" behavior in the adult literature, is associated with higher levels of patient pain behavior ${ }^{3}$ that might drive increased health service utilization.

\section{METHOD}

\section{Participants}

Participants were a subset of mothers recruited from a health maintenance organization (HMO) for a study of intergenerational transmission of illness behavior. ${ }^{4}$ The sample for the larger study $(n=449)$ included families with children between the ages of 8 and 15 years and in which no family member had a diagnosis of ulcerative colitis or Crohn disease. Approximately half of the mothers who were enrolled in the primary study had received a diagnosis of irritable bowel syndrome at a health care visit to the HMO, Group Health Cooperative of Puget Sound. Mothers participating in the larger study were sorted on a continuum from those with the highest to the lowest scores on the Protect Scale of the ARCS. Participants for the present study were recruited from the high and low ends of the continuum until at least 30 mothers were recruited for the High Protect $(n=32)$ and Low Protect $(n=35)$ groups, respectively. The purpose of recruiting from opposite ends of the distribution of Protect Scale scores was to increase the statistical power to detect an association between protect scores and other characteristics of mothers and their children. The goal of recruitment was to have at least 30 mother-child dyads in each group. To achieve this goal we sent out letters of invitation in batches, resulting in overshooting the recruitment goals. 


\section{PROCEDURE}

Mothers who participated in the original study by Levy and colleagues ${ }^{4}$ completed a research protocol that included the parent report version of the ARCS as a paper questionnaire, and their children completed a child report version of this questionnaire. These protocols were completed in their homes or at their HMO clinic, according to the family's preference. Subsequently, mothers' scores on the Protect scale were examined to identify those who scored on either end of the continuum of scores. Mothers were contacted by telephone and invited to participate in a supplemental diary study, described as a study to test the accuracy of one of the questionnaires they had completed earlier. The diary was described and mothers were told that they would be asked to complete the diary each day for 4 weeks for payment of $\$ 25$. A packet of diaries was sent to mothers by mail an average of $479.8 \pm 23.8$ days after they completed the original ARCS questionnaire assessment. Mothers were asked to return the diaries at the end of each week using a stamped return envelope provided by the investigators. They received a telephone prompt if the diaries were not returned. The refusal rate by mothers for participation in the diary study was $12.5 \%$.

Data on their child's health service utilization were obtained from HMO records for the year subsequent to mothers' completion of the ARCS questionnaire assessment. Specifically, the frequency and cost of clinic visits for abdominal symptoms and for all health care was extracted from the automated medical record.

\section{MEASURES}

\section{ARCS Questionnaire ${ }^{14}$}

The ARCS is an extension of the Illness Behavior Encouragement Scale ${ }^{7}$ which assesses solicitous parent behavior that may encourage children's illness behavior. The ARCS includes scales assessing protectiveness, minimizing, and encouraging responses to children's pain behavior. The Protect scale of the ARCS, which includes some of the items on the Illness Behavior Encouragement Scale, comprises 13 items assessing protective parent behavior in response to their children's pain behavior. The stem for each item is, "When your child has abdominal pain, how often do you ...?" Responses are rated on a 5point scale ranging from "never" (0) to "always" (4). Items refer to protective parental behavior such as giving the child special attention and limiting the child's normal activities and responsibilities. Examples include: "bring your child special treats or little gifts," "let your child stay home from school," and "give your child special privileges." A total score is obtained by calculating the average of responses to the 13 items on the scale. Alpha reliability is 0.84 . The child report version of the ARCS contains 13 items paralleling those in the adult scale and is scored in the same way. Alpha reliability for the child-report scale is 0.86 .

\section{Adult Responses to Children's Symptoms Questionnaire-Diary Version (ARCS-Diary)}

The Protect scale of the ARCS-Diary Version was created by rewording items from the ARCS to be appropriate for a Diary format. For example, the item, "How often do you do your child's chores" was reworded for the ARCS-Diary as, "Did you do your child's chores?" On each of 28 consecutive days, mothers were asked to indicate whether their child had experienced a stomach ache that day and, if so, whether she had engaged in any of the 13 protective behaviors listed. Response options were "yes" or "no." If the child had not experienced a stomach ache, the mother was directed not to complete the remaining items on the questionnaire for that day.

Total scores for protective behavior on the ARCS-Diary represented the probability of saying "yes" to each item summed across the 13 items. These probability scores were 
calculated as the number of "yes" responses to an item divided by the total number of days on which the mother indicated that her child had experienced abdominal pain. For example, if the mother reported that the child had abdominal pain on a total of 5 days and that she let the child stay home from school on 2 of those days, the probability of responding "yes" for this question would be 0.4 . If she did the child's chores for him on all 5 days, the probability for that item would be 1.0. The total score for this child's ARCS-Diary would be 0.4 plus 1.0 plus the probabilities for any other questions that were answered with one or more "yes" responses.

\section{Health Service Utilization for Abdominal Symptoms}

Group Health Cooperative maintains an automated medical record in which each health care encounter (clinic visit, hospital admission) or service (diagnostic procedures, prescriptions dispensed) is listed together with one or more ICD-9CM diagnostic codes assigned by the provider, the calculated cost of the service or visit, and the date of service. The following diagnostic codes were used to identify services for lower gastrointestinal (GI) symptoms: abdominal pain (789xx), constipation (5640), gastrointestinal motility disorder (5369), encopresis or fecal incontinence (7876), diarrhea (78791, 5645), gastroenteritis (5589), flatulence or gas (7873), abnormal bowel sounds (7875), and other symptoms involving the digestive system (7879). Costs and utilization for this aggregate category of lower gastrointestinal services were summed for the 12-month period beginning with the date of completion of the ARCS questionnaire by the child's mother. Total health care costs and total health care utilization also were calculated.

\section{DATA ANALYSIS}

Mothers and children with high and low scores on the Protect scale of the ARCS questionnaire were first compared with respect to demographic differences (Table 1). The High Protect and the Low Protect groups were then compared with respect to their ARCSDiary scores. All 67 mother-child dyads were included in this analysis. Next, the High and Low Protect groups were pooled and nonparametric Spearman correlations were computed between ARCS questionnaire scores and ARCS-Diary scores. The 27 mothers who reported that their child had no episodes of abdominal pain during the 28-day diary were eliminated from the correlation analysis because the diary measure of Protectiveness could not be computed for these subjects. Finally, the 35 High Protect subjects were compared to the 32 Low Protect subjects on health care visits and health care costs.

For comparisons between groups, $t$ tests were used for normally distributed continuous variables such as age, $\chi^{2}$ was used for frequency data, and nonparametric Mann-Whitney $U$ test statistics were used for health care utilization and health care cost data (because these data were skewed, with many subjects reporting no GI-related health care visits or costs). Test statistics with associated probabilities less than or equal to 0.05 were interpreted as statistically significant.

In a secondary analysis we sought to determine whether differences between the High Protect and Low Protect groups on health care utilization and cost data remained significant after adjusting for demographic differences between groups; the General Linear Models statistical program ${ }^{17}$ was used for this purpose. For these analyses, the health care utilization and cost data were log-transformed to reduce skew, and mother's age, education, employment status, and race were entered as covariates. 


\section{RESULTS}

Child age was similar in the High and Low Protect groups (11.66 $\pm 3.02 \mathrm{y}$ in the Low group and $11.06 \pm 2.60 \mathrm{y}$ in the High group). There were more girls in the Low Protect group than in the High Protect group (53\% vs. 37\%), but this difference was not statistically significant $\left(\chi^{2}=1.73, P=0.189\right)$. Demographic differences for the mothers are shown in Table 1 . The High Protect mothers tended to be younger and to have discontinued their education earlier. High Protect mothers were significantly less likely to be whites.

The majority of participants ( 63 of $67,94.0 \%$ ) completed all 28 days of diary entries. The data were examined to determine whether the High and Low Protect groups differed in the number of diary days on which mothers reported that their children had an episode of abdominal pain. There was no significant difference in the average number of days with pain during the 28-day diary study $(1.88 \pm 3.28 \mathrm{~d}$ for Low vs. $2.29 \pm 2.67 \mathrm{~d}$ for High, NS). In the Low Protect group, $50.0 \%$ had one or more days with a pain episode and $12.5 \%$ had 5 or more days with pain episodes. In the High Protect group, 68.6\% had one or more days with a pain episode and $17.1 \%$ had 5 or more days with pain episodes.

\section{Parent report on the ARCS compared to Diary Data}

As predicted, the High Protect group had significantly higher scores than the Low Protect group on the ARCS-Diary $(6.60 \pm 4.04$ vs. $1.13 \pm 0.82, P<0.001)$. A Spearman rank-order correlation was computed to compare mothers' scores on the ARCS questionnaire to their subsequent scores on the ARCS-Diary. This correlation was limited to the 40 mothers whose children had abdominal pain on at least one day during the 28 days of the diary study (16 subjects in the Low Protect group and 24 in the High Protect group). The correlation was significant with $\rho=0.614(P<0.001)$.

\section{Child-report form of the ARCS}

The High and Low Protect mother-child dyads, who had been selected for inclusion based on the ARCS scores of the mothers, were also different on the child report form of the ARCS Protect scale: $29.22 \pm 11.49$ versus $22.45 \pm 11.17$ for the High and Low Protect groups respectively, $t=2.37, P=0.01$. Child ARCS Protect scores were available for 31 in the Low Protect group and 32 in the High Protect group. The correlation between scores of mothers and scores of children on the Protect scale was $\rho=0.334, P=0.007$. However, scores on the child report form of the Protect scale did not correlate significantly with the mother-report ARCS-diary scores $(\rho=0.141, \mathrm{NS})$.

\section{Health care utilization and costs}

HMO medical records for the year subsequent to baseline questionnaire assessment indicated that children of mothers in the High Protect group made significantly $(Z=1.956, P$ $=0.05)$ more GI visits $(0.14 \pm 0.43)$ compared to children of mothers in the Low Protect group (no visits), and they incurred significantly $(Z=1.956, P=0.05)$ higher GI-related health care costs $(\$ 16.47 \pm \$ 53.18$ per year) compared to children of mothers in the Low Protect group, whose children had no GI-related health care costs. There were no significant differences between the groups in the total number of ambulatory health care visits $(3.74 \pm$ 2.87 vs. $4.78 \pm 7.27$ for High vs. Low Protect groups, NS) nor total health care costs ( $\$ 946.42 \pm \$ 1198.46$ vs. $\$ 1664.87 \pm \$ 4085.43$ for High vs. Low Protect groups, NS).

A secondary analysis was used to determine whether the differences in health care cost and utilization were attributable to demographic differences between the High Protect mothers and the Low Protect groups. Adjusting for the mother's age, education, employment status, and race attenuated the differences between groups for both the number of GI health care 
visits $[F(1,55)=2.02, P=0.16]$ and the GI health care costs $[F(1,55)=2.099, P=0.15]$. For both comparisons, the only significant covariate was mother's education, with higher education associated with lower costs and number of visits.

\section{DISCUSSION}

These data demonstrate that mothers' reports of the extent to which they are usually protective in response to their children's pain complaints, assessed by the ARCS questionnaire, are strongly associated with their reports of protective responses to specific instances of their children's pain complaints during a month of daily diary assessments. Furthermore, mothers who obtained high scores on the Protect scale of the ARCS made significantly more HMO clinic visits and had higher health care costs for their children's abdominal symptoms during the subsequent year than mothers who obtained low scores on the Protect Scale of the ARCS. These findings demonstrate the stability of parental responses to their children's abdominal pain over time and support the validity of the ARCS questionnaire.

The child report version of the ARCS showed a significant but modest correlation with the parent report ARCS $(\rho=0.338, P=0.007)$, but did not correlate significantly with the parent-report ARCS-diary $(\rho=0.141, \mathrm{NS})$. We conclude that the parent report version of the ARCS more accurately reflects the behaviors of parents in response to children's somatic complaints. We speculate that children, especially young children, may have difficulty critically evaluating the behavior of their parents and/or they may have difficulty with the level of abstraction demanded by the ARCS.

When the demographic characteristics of the mothers in the High and Low Protect groups were compared, we observed that the mothers in the High Protect groups were more likely to come from ethnic minorities (especially Asian and Hispanic), and they tended to be younger and to have terminated their education earlier. These differences suggest that there are cultural differences in the ways parents respond to the somatic complaints of their children, including what behaviors they consider to be normal and appropriate. The data also suggest that there may be socioeconomic differences in the ways mothers respond to their children's somatic complaints. These demographic differences must be considered when interpreting observed differences between parents in their responses to somatic complaints. Further research is needed to understand these cultural and socioeconomic associations.

The use of small samples is a limitation of this study. The sample size was sufficient to achieve the primary aim of testing the agreement between parent responses on the ARCS questionnaire and their subsequent responses on a daily diary. However, larger samples, including children with more frequent pain, would have made it possible to explore the relation of ARCS responses to child characteristics including sex and pain frequency. A second limitation was the relatively long interval between the completion of the ARCS questionnaire and the diary study. Changes in parent behavior or in the child's health status between these 2 intervals may have contributed to measurement error. However, this long interval has the advantage of showing that behaviors of mothers toward their children's abdominal pain complaints are relatively stable over time.

In addition to the support it provides for the validity of the parent report version of the ARCS, the study makes 2 important substantive contributions to the empirical literature on parental responses to children's pain. First, the study is based on daily reports of behavior from a community sample of mothers who reported their responses to a pain complaint that is common even among healthy schoolchildren. ${ }^{16}$ Previous research in this area has been conducted in clinical settings with pediatric patients with chronic or acute procedural pain. 
Thus, results of this study suggest that it is possible to make meaningful discriminations between parents with different levels of protective responding to children's everyday pain complaints in a nonclinical setting, and that this behavior is relatively stable during the course of a year.

Second, the study showed that mothers' reports of protective responding to their children's abdominal complaints at home predicted subsequent health service utilization when their children had abdominal complaints. This finding underscores that the tendency to respond in a protective way to children's pain may be associated with a variety of parent behaviors ranging from relieving the child from chores at home, to taking the child to a medical clinic. Additional work is needed to extend this research to examine the effects of culture and sex on parents' protective behavior.

\section{Acknowledgments}

Supported by NIH grants RO1 HD36069, RO1 DK31369, and R24 DK67674.

\section{REFERENCES}

1. Itkowitz NI, Kerns RD, Otis JD. Support and coronary heart disease: the importance of significant other responses. J Behav Med. 2003; 26:19-30. [PubMed: 12690944]

2. Lousberg R, Schmidt AJM, Groenman NH. The relationship between spouse solicitousness and pain behavior: searching for more experimental evidence. Pain. 1992; 51:75-79. [PubMed: 1454408]

3. Romano JM, Turner JA, Jensen MP, et al. Chronic pain patient-spouse behavioral interactions predict patient disability. Pain. 1995; 63:353-360. [PubMed: 8719536]

4. Levy RL, Whitehead WE, Walker LW, et al. Increased somatic complaints and health care utilization in children: effects of parent IBS status and parent response to gastrointestinal symptoms. Am J Gastroenterol. 2004; 99:2442-2451. [PubMed: 15571594]

5. Walker LS, Claar RL, Garber J. Social consequences of children's pain: when do they encourage symptom maintenance? J Pediatr Psychol. 2002; 27:689-698. [PubMed: 12403859]

6. Walker LS, Williams SE, Smith CA, et al. Parent attention versus distraction: impact on symptom complaints by children with and without chronic functional abdominal pain. Pain. 2006:71.

7. Walker LS, Zeman JL. Parental response to child illness behavior. J Pediatr Psychol. 1992; 17:49_ 71. [PubMed: 1545321]

8. Gidron Y, McGrath PJ, Goodday R. The physical and psychosocial predictors of adolescents' recovery from oral surgery. J Behav Med. 1995; 18:385-399. [PubMed: 7500329]

9. Fordyce, WE. Behavioral Methods for Chronic Pain and Illness. Mosby; St Louis: 1976.

10. Kerns RD, Rosenberg R. Pain-relevant responses from significant others: development of a significant-other version of the WHYMPI scales. Pain. 1995; 61:245-249. [PubMed: 7659434]

11. Marvan ML, Cortes-Iniestra S. Women's beliefs about the prevalence of premenstrual syndrome and biases in recall of premenstrual changes. Health Psychol. 2001; 20:276-280. [PubMed: 11515739]

12. Tourangeau, R. Remembering what happened: memory errors and survey reports. In: Stone, AA.; Turkkan, JS.; Bachrach, CA., et al., editors. The Science of Self-report: Implications for Research and Practice. Lawrence Erlbaum Associates, Inc; 1999. p. 29-47.

13. Fillligim RB, Doleys DM, Edwards RR, et al. Spousal responses are differentially associated with clinical variables in women and men with chronic pain. Clin J Pain. 2003; 19:217-224. [PubMed: 12840615]

14. Van Slyke DA, Walker LS. Mothers' responses to children's pain. Clin J Pain. In press.

15. Keefe, FJ. Self report of pain: issues and opportunities. In: Stone, AA.; Turkkan, JS.; Bachrach, CA., et al., editors. The Science of Self-report: Implications for Research and Practice. Lawrence Erlbaum Associates, Inc; 1999. p. 317-337. 
16. McGrath, PA. Pain in Children: Nature, Assessment, and Treatment. Guilford Press; New York: 1990.

17. SPSS, Inc. General Linear Models. Jan 9. 200413.0 for Windows233 South Wacker Drive, 11th Floor, Chicago, IL 60606

18. Drossman, DA.; Corazziari, E.; Talley, NJ., et al. The Functional Gastrointestinal Disorders. 1st ed.. Little, Brown; Boston: 1994.

19. Kerns RD, Southwick S, Giller EL, et al. The relationship between reports of pain-related social interactions and expressions of pain and affective distress. Behav Ther. 1991; 22:101-111.

20. Kerns RD, Turk DC, Rudy TE. The West Haven-Yale Multi-dimensional Pain Inventory (WHYMPI). Pain. 1985; 23:345-356. [PubMed: 4088697]

21. Whitehead WE, Crowell MD, Heller BR, et al. Modeling and reinforcement of the sick role during childhood predicts adult illness behavior. Psychosom Med. 1994; 56:541-550. [PubMed: 7871110]

22. Whitehead WE, Busch CM, Heller BR, et al. Social learning influences on menstrual symptoms and illness behavior. Health Psychol. 1986; 5:13-23. [PubMed: 3720717] 
